\title{
SUPPLYMENTARY INFORMATION
}

\section{Probing polymer chain folding in solution using second harmonic light scattering}

Ramkrishna Sarkar, ${ }^{a}$ Kamini Mishra, ${ }^{\mathrm{b}}$ Harshita ${ }^{\mathrm{a}}$, Puspendu Kumar Das ${ }^{\mathrm{a}}$ and S. Ramakrishnan*a

aDepartment of Inorganic and Physical Chemistry, Indian Institute of Science, Bangalore 560012, INDIA

${ }^{\mathrm{b}}$ Department of Chemistry, School of Advanced Sciences, Vellore Institute of Technology, Vellore 632 014, Tamil Nadu, INDIA.

e-mail: raman@iisc.ac.in

\section{$\underline{\text { Table of contents }}$}

\begin{tabular}{|c|c|}
\hline Title & Page number \\
\hline $\begin{array}{c}\text { Synthesis of intermediates and } \\
\text { monomers }\end{array}$ & S2-S10 \\
\hline Figure S1 & S10 \\
\hline Figure S2 & S11 \\
\hline Figure S3 & S12 \\
\hline Figure S4 & S12 \\
\hline Figure S5 & S13 \\
\hline Figure S6 & S14 \\
\hline Figure S7 & S15 \\
\hline Figure S8 & S15 \\
\hline Figure S9 & S16 \\
\hline Table S1 & S16 \\
\hline Table S2 & S17 \\
\hline Estimation of $f$ (local field factor) & S17 \\
\hline
\end{tabular}


The propargyl alcohol, diethyl-2-methyl-2propargylmalonate (1), 2-methyl-2propargylmalonic acid (2), 15-bromopentadecanoic acid, 15-bromo-t-butylpentadecanoate (3), 15-bromopentadecanol (6), PEG-thiol were synthesized following the procedure reported earlier. ${ }^{1,2}$

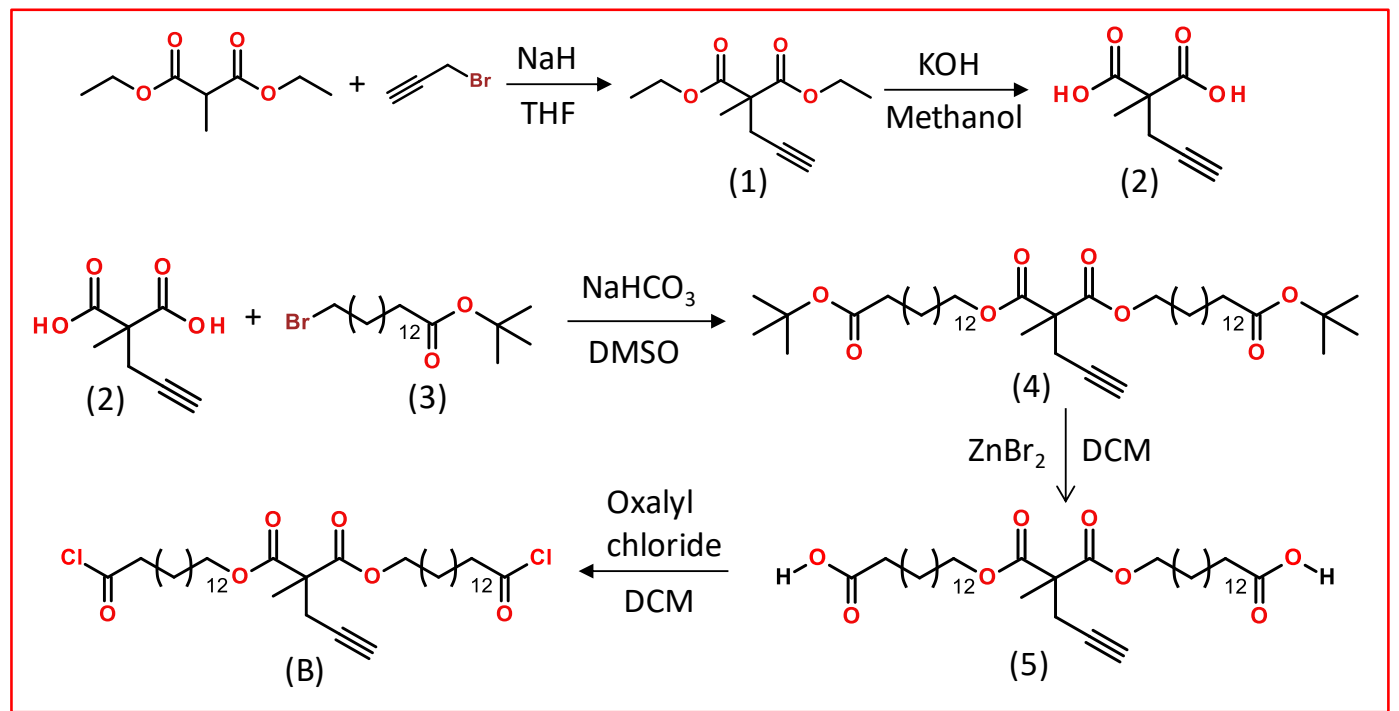

Scheme S1: Synthetic scheme of propargyl bearing diacid chloride monomer (B)

\section{Di(w-t-butylpentadecanoate) 2- methyl-2- propargylmalonate (4)}

$2.76 \mathrm{~g}$ (17.83 mmol) 2-Methyl-2-propargylmalonic (2) acid and $9.12 \mathrm{~g}$ (108.6 mmol) sodium bicarbonate were taken in $100 \mathrm{~mL}$ DMF. $16 \mathrm{~g}$ (42.44 mmol) 15-bromo-t-butylpentadecanoate (3) along with catalytic amount potassium iodide were added to it. The resulted reaction mixture was stirred at $45{ }^{\circ} \mathrm{C}$ for 4 days. $150 \mathrm{~mL}$ ethyl acetate was added to it and DMF was extracted in $450 \mathrm{~mL}(3 \times 150 \mathrm{~mL}) 1 \mathrm{wt}$. \% aqueous solution of lithium chloride. Organic layer was dried over sodium sulphate and concentrated to yield final product. The crude product was with $85 \%$ yield $(11.26 \mathrm{~g})$ utilized for the next step of conversion.

\section{Di(w-pentadecanoate) 2- methyl-2-propargylmalonate (5)}

$8 \mathrm{~g}(10.68 \mathrm{mmol})$ of compound 4 and $9.6 \mathrm{~g}$ (42.72 mmol) zinc bromide were taken in $250 \mathrm{~mL}$ round bottom flask fitted with calcium chloride guard tube. $50 \mathrm{~mL}$ dry DCM was added to the mixture and stirred at room temperature for $24 \mathrm{~h} .100 \mathrm{ml}$ chloroform was added to it and organic solvent containing product was washed twice $(2 \times 100 \mathrm{~mL})$ with water and dried over sodium sulfate. Removal of solvent under reduced pressure yielded the desired diacid (5) as a white solid which was purified further by reprecipitation form methanol with $55 \%(3.74 \mathrm{~g})$ 
yield. The acid chloride monomer (B) was prepared from diacid 5 prior to polymerization using oxalyl chloride.

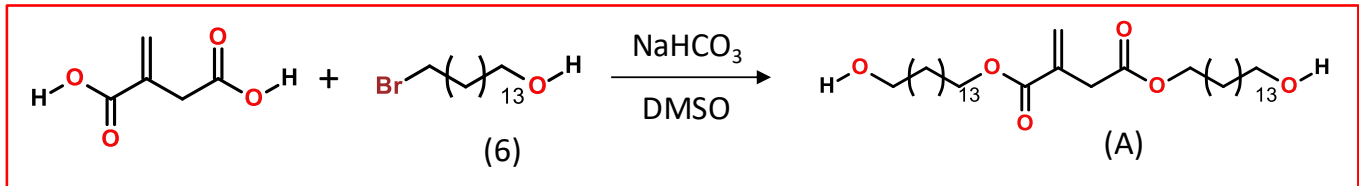

Scheme S2: Synthetic scheme of the itaconate bearing diol (A).

\section{Di (w-hydroxypentadecanyl) itaconate (A)}

$3 \mathrm{~g}(23.07 \mathrm{mmol})$ itaconic acid and $11.63 \mathrm{~g}(138.5 \mathrm{mmol})$ sodium bicarbonate were taken in $100 \mathrm{~mL}$ DMF. $16.94 \mathrm{~g}$ (55.36 mmol) 15-bromopentadecanol was added to it. The resulted reaction mixture was stirred at $45{ }^{\circ} \mathrm{C}$ for 4 days. $150 \mathrm{~mL}$ chloroform was added to it and DMF was extracted in $450 \mathrm{~mL}(3 \times 150 \mathrm{~mL}) 1 \mathrm{wt}$. \% aqueous solution of lithium chloride. Organic layer was dried over sodium sulfate and concentrated to yield final product. The crude product was first recrystallized from cold methanol with $52 \%$ yield $(6.98 \mathrm{~g})$

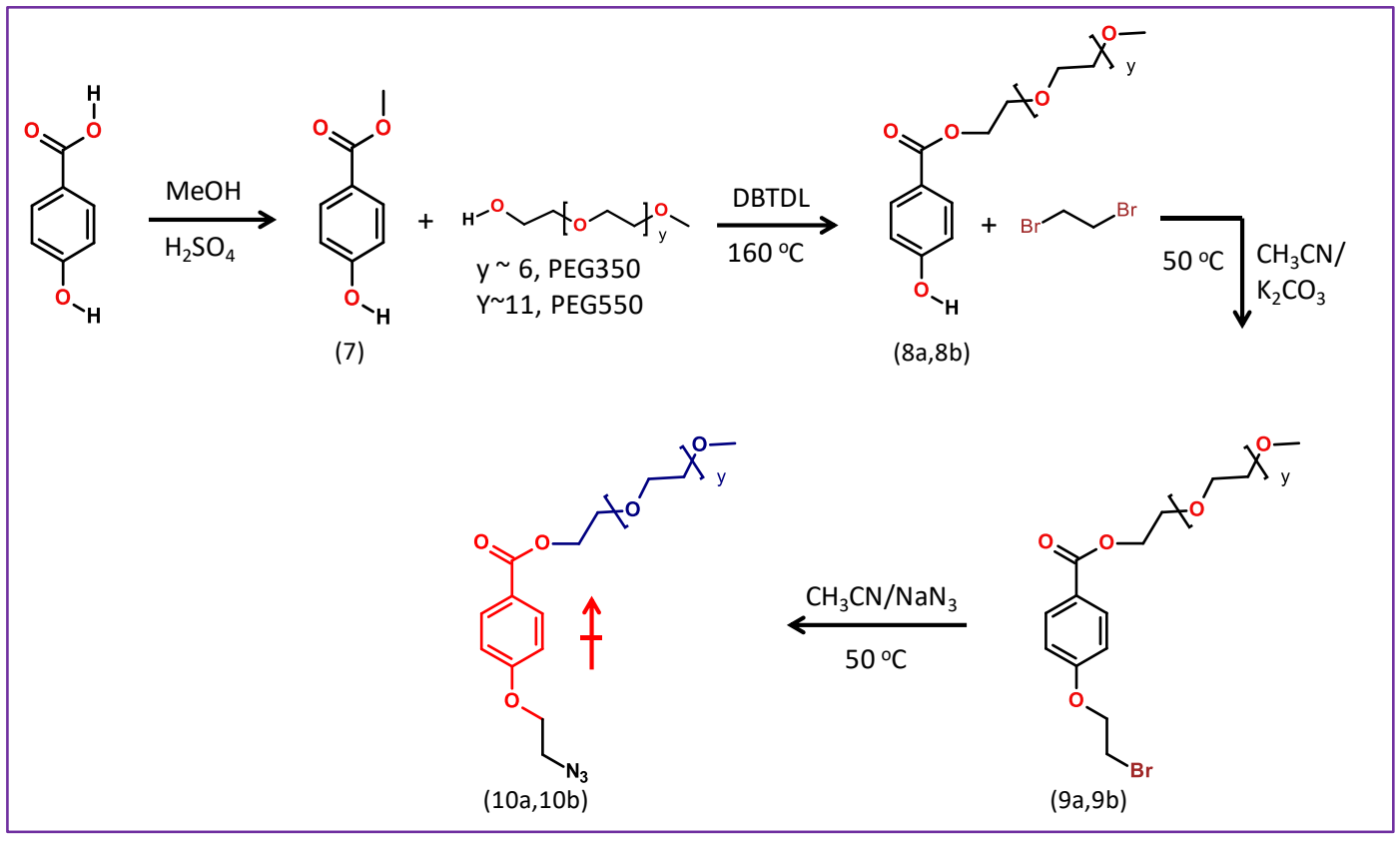

Scheme S3: Synthetic scheme of chromophore bearing clickable PEG azides.

\section{Methyl-4-hydroxybenzoate (7)}

$5.5 \mathrm{~g}$ ( $36.23 \mathrm{mmol}$ ) 4-hydoxy benzoic acid and $100 \mathrm{ml}$ dry methanol were taken in 250 round bottom flask. $1 \mathrm{ml}$ concentrated sulfuric acid was added to it and reaction mixture was reflux for overnight. Subsequently, sodium bicarbonate was added for the neutralization of acid. 
Methanol containing product was filtered and concentrated to yield product with $88 \%$ yield (4.95 g).

${ }^{1} \mathrm{H} \operatorname{NMR}(\delta, \mathrm{ppm}, \mathrm{DMSO}): 7.96$ (d, 2H, $\left.\mathrm{HO}-\mathrm{Ar}-\mathrm{CO}_{2} \mathrm{CH}_{3}\right) ; 7.39$ (s, 1H, $\left.\mathrm{HOArCO}{ }_{2} \mathrm{CH}_{3}\right) ; 6.93(\mathrm{~d}, 2 \mathrm{H}$, $\left.\operatorname{Ar}-\mathrm{CO}_{2} \mathrm{CH}_{3}\right) ; 3.93\left(\mathrm{~s}, 3 \mathrm{H}, \mathrm{Ar}-\mathrm{CO}_{2} \mathrm{CH}_{3}\right)$

\section{PEG-4-hydroxybenzoate $(8 \mathrm{a}, 8 \mathrm{~b})$}

$2.2 \mathrm{~g}$ (14.46 mmol) methyl-4-hydroxybenzoate (7) and $17.35 \mathrm{mmol}$ of PEG350/PEG550 monomethyl ether were placed in Kugelrohr along with $30 \mathrm{mg}(4.38 \mathrm{mmol})$ DBTDL. The trasesterification was carried out at $160^{\circ} \mathrm{C}$ for $8 \mathrm{~h}$ at $400 \mathrm{mbar}$ vacuum. The obtained product containing a little excess unreacted PEG was used directly for next step of transformation.

${ }^{1} \mathrm{H} \operatorname{NMR}\left(\delta, p p m, \mathrm{CDCl}_{3}\right): 7.93\left\{\mathrm{~d}, 2 \mathrm{H}, \mathrm{ArCO}_{2}-\left(\mathrm{CH}_{2} \mathrm{CH}_{2} \mathrm{O}\right)_{\mathrm{n}} \mathrm{CH}_{3}\right\} ; 6.88\left\{\mathrm{~d}, 2 \mathrm{H}, \mathrm{ArCO}_{2}-\left(\mathrm{CH}_{2} \mathrm{CH}_{2} \mathrm{O}\right)_{\mathrm{n}}\right.$ $\left.\mathrm{CH}_{3}\right\} ; 4.42\left\{\mathrm{t}, 2 \mathrm{H}, \quad \mathrm{ArCO}_{2}-\mathrm{CH}_{2} \mathrm{CH}_{2} \mathrm{O}\left(\mathrm{CH}_{2} \mathrm{CH}_{2} \mathrm{O}\right)_{\mathrm{n}-1} \mathrm{CH}_{3}\right\} ; 3.6-3.7\left\{\mathrm{~m}, 30 \mathrm{H}, \mathrm{ArCO}_{2}-\right.$ $\left.\mathrm{CH}_{2} \mathrm{CH}_{2} \mathrm{O}\left(\mathrm{CH}_{2} \mathrm{CH}_{2} \mathrm{O}\right)_{n-1} \mathrm{CH}_{3}\right\} ; 3.36\left\{\mathrm{~s}, 3 \mathrm{H}, \mathrm{ArCO}_{2}-\mathrm{CH}_{2} \mathrm{CH}_{2} \mathrm{O}\left(\mathrm{CH}_{2} \mathrm{CH}_{2} \mathrm{O}\right)_{n-1} \mathrm{CH}_{3}\right\}$

\section{PEG-4-(2-bromoethyloxy) benzoate (9a, 9b)}

$8 \mathrm{mmol}$ PEG-4-hydroxy benzoate $(8 \mathrm{a}, 8 \mathrm{~b})$ and $4.416 \mathrm{~g}(32 \mathrm{mmol})$ potassium carbonate were taken in $250 \mathrm{ml}$ round bottom flask along with $100 \mathrm{ml}$ acetonitrile. $12 \mathrm{~g}$ (64.2 mmol) 1,2dibromoethane was added to it. The reaction carried out at $50{ }^{\circ} \mathrm{C}$ for $72 \mathrm{~h}$ under inert atmosphere. Subsequently, acetonitrile was removed, and $100 \mathrm{ml}$ water was added to it. The product was extracted in $150 \mathrm{ml}(3 \times 50 \mathrm{ml})$ ethyl acetate and dried over sodium sulfate. Finally, product was obtained by removing organic solvent under reduced pressure with nearly $70 \%$ yield.

${ }^{1} \mathrm{H} \operatorname{NMR}\left(\delta, \mathrm{ppm}, \mathrm{CDCl}_{3}\right): 8.01\left\{\mathrm{~d}, 2 \mathrm{H}, \mathrm{BrCH}_{2} \mathrm{CH}_{2}-\mathrm{OAr}-\mathrm{CO}_{2}-\left(\mathrm{CH}_{2} \mathrm{CH}_{2} \mathrm{O}\right)_{\mathrm{n}} \mathrm{CH}_{3}\right\} ; 6.91\{\mathrm{~d}, 2 \mathrm{H}$, $\left.\mathrm{BrCH}_{2} \mathrm{CH}_{2} \mathrm{OArCO}_{2}-\left(\mathrm{CH}_{2} \mathrm{CH}_{2} \mathrm{O}\right)_{n} \mathrm{CH}_{3}\right\} ; 4.44\left\{\mathrm{t}, 2 \mathrm{H}, \mathrm{BrCH} \mathrm{CH}_{2} \mathrm{O}-\mathrm{Ar}-\mathrm{CO}_{2}-\mathrm{CH}_{2} \mathrm{CH}_{2} \mathrm{O}\left(\mathrm{CH}_{2} \mathrm{CH}_{2} \mathrm{O}\right)_{n-1}\right.$ $\left.\mathrm{CH}_{3}\right\} ; \quad 4.32\left\{\mathrm{q}, 2 \mathrm{H}, \quad \mathrm{BrCH}{ }_{2} \mathrm{CH}_{2} \mathrm{O}-\mathrm{Ar}-\mathrm{CO}_{2}-\left(\mathrm{CH}_{2} \mathrm{CH}_{2} \mathrm{O}\right)_{\mathrm{n}} \mathrm{CH}_{3}\right\} ;$ 3.6-3.7 $\left\{\mathrm{m}, \mathrm{ArCO}_{2}-\mathrm{CH}_{2} \mathrm{CH}_{2} \mathrm{O}\right.$ $\left.\left(\mathrm{CH}_{2} \mathrm{CH}_{2} \mathrm{O}\right)_{\mathrm{n}-1} \mathrm{CH}_{3}\right\} ; 3.36\left\{\mathrm{~s}, 3 \mathrm{H}, \mathrm{ArCO}_{2}\left(\mathrm{CH}_{2} \mathrm{CH}_{2} \mathrm{O}\right)_{n} \mathrm{CH}_{3}\right\}$

\section{PEG-4-(2-azidoethyloxy) benzoate $(10 \mathrm{a}, 10 \mathrm{~b})$}

7 mmol PEG-4-(2-bromoethyloxy) benzoate $(9 \mathrm{a}, 9 \mathrm{~b})$ and $0.96 \mathrm{~g}$ sodium azide (14.8 mmol) taken in $100 \mathrm{ml}$ round bottom flask and $50 \mathrm{ml}$ acetonitrile was added to it. The reaction was carried out at $50^{\circ} \mathrm{C}$ for $72 \mathrm{~h}$ under inert atmosphere. Subsequently, acetonitrile was removed, 
and $50 \mathrm{ml}$ water was added to it. The product was extracted in $120 \mathrm{ml}$ ( $3 \times 40 \mathrm{ml}$ ) ethyl acetate and dried over sodium sulfate. Finally, product was obtained by removing organic solvent under reduced pressure with $80 \%$ yield.

${ }^{1} \mathrm{H} \operatorname{NMR}\left(\delta, \mathrm{ppm}, \mathrm{CDCl}_{3}\right): 8\left\{\mathrm{~d}, 2 \mathrm{H}, \mathrm{N}_{3} \mathrm{CH}_{2} \mathrm{CH}_{2} \mathrm{OAr}-\mathrm{CO}_{2}-\left(\mathrm{CH}_{2} \mathrm{CH}_{2} \mathrm{O}\right)_{\mathrm{n}} \mathrm{CH}_{3}\right\} ; 6.92\{\mathrm{~d}, 2 \mathrm{H}$, $\left.\mathrm{N}_{3} \mathrm{CH}_{2} \mathrm{CH}_{2} \mathrm{OArCO}_{2}-\left(\mathrm{CH}_{2} \mathrm{CH}_{2} \mathrm{O}\right)_{n} \mathrm{CH}_{3}\right\} ; 4.44\left\{\mathrm{t}, 2 \mathrm{H}, \mathrm{N}_{3} \mathrm{CH}_{2} \mathrm{CH}_{2} \mathrm{O}-\mathrm{Ar}-\mathrm{CO}_{2}-\mathrm{CH}_{2} \mathrm{CH}_{2} \mathrm{O}\left(\mathrm{CH}_{2} \mathrm{CH}_{2} \mathrm{O}\right)_{n-1}\right.$ $\left.\mathrm{CH}_{3}\right\} ; \quad 4.20\left\{\mathrm{t}, 2 \mathrm{H}, \quad \mathrm{N}_{3} \mathrm{CH}_{2} \mathrm{CH}_{2} \mathrm{O}-\mathrm{Ar}-\mathrm{CO}_{2}-\left(\mathrm{CH}_{2} \mathrm{OCH}_{2} \mathrm{O}\right)_{\mathrm{n}} \mathrm{CH}_{3}\right\} ;$ 3.6-3.7 \{m, $\operatorname{ArCO}_{2-}$ $\left.\mathrm{CH}_{2} \mathrm{CH}_{2} \mathrm{O}\left(\mathrm{CH}_{2} \mathrm{CH}_{2} \mathrm{O}\right)_{n-1} \mathrm{CH}_{3}\right\} ; 3.36\left\{\mathrm{~s}, 3 \mathrm{H}, \mathrm{ArCO}_{2}-\mathrm{CH}_{2} \mathrm{CH}_{2} \mathrm{O}\left(\mathrm{CH}_{2} \mathrm{CH}_{2} \mathrm{O}\right)_{n-1} \mathrm{CH}_{3}\right\}$;

\section{Model chromophore: Methyl-4-methoxybenzoate}

$2 \mathrm{~g}$ (13.16 mmol) Methyl-4-hydroxybenzoate (1) and $7.26 \mathrm{~g}$ (52.64 mmol) potassium carbonate were taken in $50 \mathrm{ml}$ acetonitrile. $5.60 \mathrm{~g}$ (39.47 mmol) methyl iodide was added to it. The reaction carried out at $50{ }^{\circ} \mathrm{C}$ for $72 \mathrm{~h}$ under inert atmosphere. Subsequently, acetonitrile was removed, and $100 \mathrm{ml}$ water was added to it. The product was extracted in $120 \mathrm{ml}$ (3x40 ml) ethyl acetate and dried over sodium sulfate. Finally, product was obtained by removing organic solvent under reduced pressure with $89 \%$ yield (1.94 g).

${ }^{1} \mathrm{H} \operatorname{NMR}\left(\delta, p p m, \mathrm{CDCl}_{3}\right): 7.98\left(\mathrm{~d}, 2 \mathrm{H}, \mathrm{CH}_{3} \mathrm{OArCO}{ }_{2} \mathrm{CH}_{3}\right) ; 6.90\left(\mathrm{~d}, 2 \mathrm{H}, \mathrm{CH}_{3} \mathrm{OArCO} \mathrm{CH}_{3}\right) ; 3.88$ (s, $\left.3 \mathrm{H}, \mathrm{CH}_{3} \mathrm{OArCO}{ }_{2} \mathrm{CH}_{3}\right) ; 3.85\left(\mathrm{~s}, 3 \mathrm{H}, \mathrm{CH}_{3} \mathrm{OArCO} \mathrm{CH}_{3}\right)$

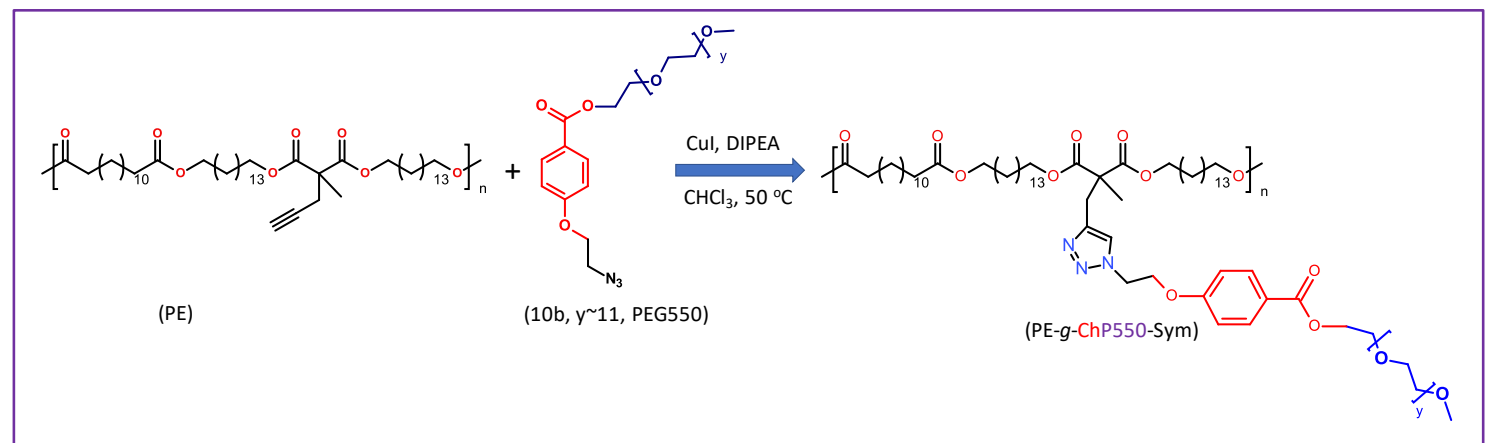

Scheme 4: Synthetic scheme for the model symmetrical polymer, (PE-g-ChP550-Sym) with PEG 550 segments.

Propargyl bearing clickable parent polyester (PE) was synthesized using our previously reported synthetic procedures. ${ }^{2}$ 


\section{Synthesis of PE-g-ChP550-Sym}

$0.2 \mathrm{~g}(0.244 \mathrm{mmol})$ of parent polyester (PE) and $0.3 \mathrm{~g}(0.375 \mathrm{mmol})$ of chromophore bearing PEG550 azide (10b) were dissolved in $5 \mathrm{~mL}$ chloroform. $10 \mathrm{~mol} \%$ copper (I) iodide and 1 equivalent diisopropyl ethylamine (DIPEA) were added to it. The mixture was purged with nitrogen for $15 \mathrm{~min}$, tightly sealed and maintained at $50^{\circ} \mathrm{C}$ for 3 days with continuous stirring. Subsequently, the reaction mixture was centrifuged to remove the copper salt. The chromophore clicked polymers (PE-g-ChP550-sym) was purified by reprecipitation (twice) using chloroform as a solvent and methanol as the nonsolvent. Finally, the polymer samples were dried under reduced pressure to give around $77 \%$ yield.

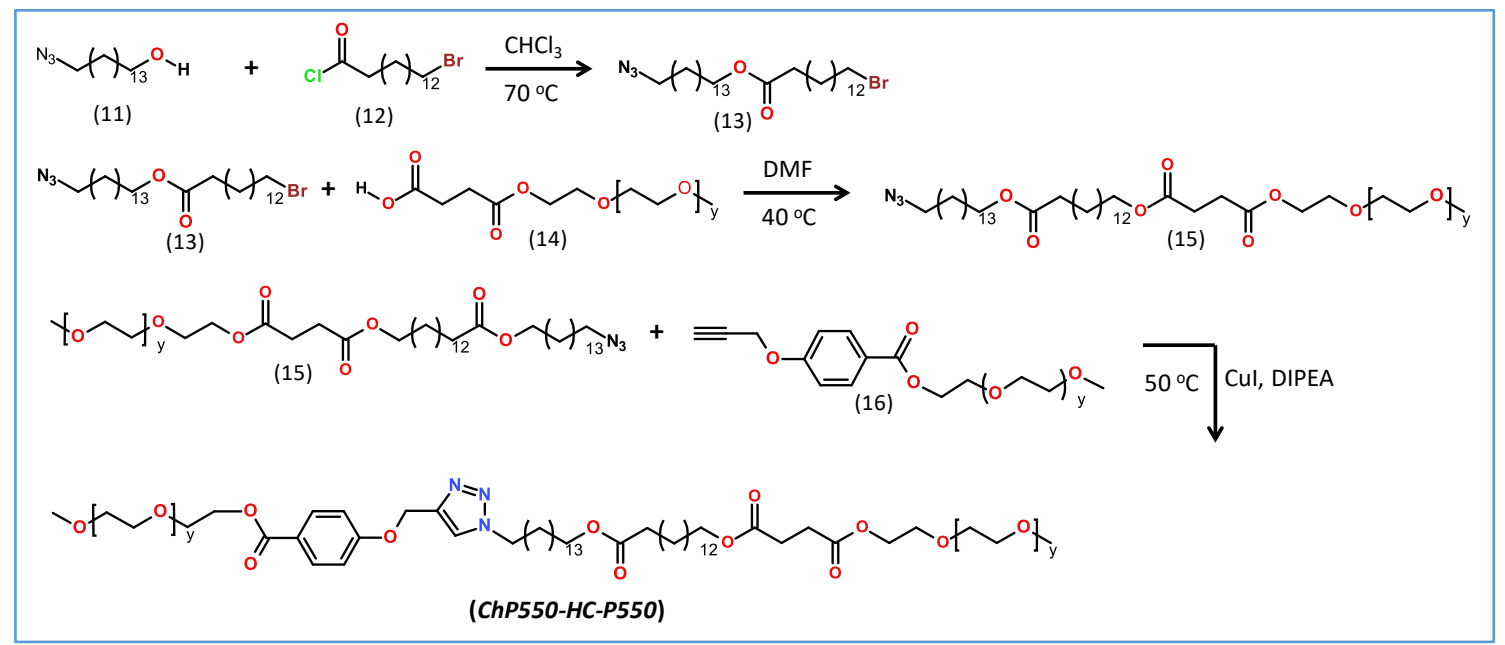

Scheme 5: Synthesis scheme of the chromophore-bearing bola-amphiphilic model compound, ChP550HC-P550.

\section{5-Azidopentadecan-1-ol (11)}

$3 \mathrm{~g}$ 15-bromopentadecan-1-ol $(9.8 \mathrm{mmol})$ and $2.5 \mathrm{~g}$ sodium azide $(38.46 \mathrm{mmol})$ taken in 100 $\mathrm{ml}$ round bottom flask and $50 \mathrm{ml}$ acetonitrile was added to it. The reaction was carried out at $50{ }^{\circ} \mathrm{C}$ for $72 \mathrm{~h}$ under inert atmosphere. Subsequently, acetonitrile containing product was filtered and dried over sodium sulfate. Finally, product was obtained by removing organic solvent under reduced pressure with $87 \%$ yield $(2.29 \mathrm{~g})$.

${ }^{1} \mathrm{HNMR}\left(\delta, \mathrm{ppm}, \mathrm{CDCl}_{3}\right): 3.63\left\{\mathrm{t}, 2 \mathrm{H}, \mathrm{HOCH}_{2} \mathrm{CH}_{2}\left(\mathrm{CH}_{2}\right){ }_{11} \mathrm{CH}_{2} \mathrm{CH}_{2} \mathrm{~N}_{3}\right\} ; 3.52\left\{\mathrm{t}, 2 \mathrm{H}, \mathrm{HOCH}_{2} \mathrm{CH}_{2}\right.$ $\left.\left(\mathrm{CH}_{2}\right)_{11} \mathrm{CH}_{2} \mathrm{CH}_{2} \mathrm{~N}_{3}\right\} ; 1.56\left\{\mathrm{~m}, 4 \mathrm{H}, \mathrm{HOCH}_{2} \mathrm{CH}_{2}\left(\mathrm{CH}_{2}\right)_{11} \mathrm{CH}_{2} \mathrm{CH}_{2} \mathrm{~N}_{3}, \mathrm{HOCH}_{2} \mathrm{CH}_{2}\left(\mathrm{CH}_{2}\right)_{11} \mathrm{CH}_{2} \mathrm{CH}_{2} \mathrm{~N}_{3}\right\}$ 


\section{Compound 13}

$1.8 \mathrm{~g}$ 15-bromopentadecanoic acid $(4.67 \mathrm{mmol})$ taken in $50 \mathrm{ml}$ round bottom flask and $20 \mathrm{ml}$ dry DCM was added to it. $2.1 \mathrm{~g}(16.67 \mathrm{mmol})$ oxalyl chloride and capillary drop DMF were added to it. The reaction was carried out at $40{ }^{\circ} \mathrm{C}$ for $5 \mathrm{~h}$ under calcium chloride guard tube. Subsequently, removal of solvent and excess oxalyl chloride under vacuum yielded the quantitative amount 15-bromopentadecanoyl chloride (12). The in situ prepared acid chloride was (12) was dissolved in $20 \mathrm{ml}$ chloroform and $1.5 \mathrm{~g}$ (4.67 mmol) 15-azidopentadecan-1-ol (11) was added to it and heated at $70{ }^{\circ} \mathrm{C}$ for $12 \mathrm{~h}$. The product was obtained by removing chloroform under reduced pressure and recrystallized from methanol to yield $1.91 \mathrm{~g}$ product (58\% yield).

${ }^{1} \mathrm{HNMR}\left(\delta, \mathrm{ppm}, \mathrm{CDCl}_{3}\right): 4.07\left\{\mathrm{t}, 2 \mathrm{H}, \mathrm{BrCH}_{2} \mathrm{CH}_{2}\left(\mathrm{CH}_{2}\right){ }_{11} \mathrm{CH}_{2} \mathrm{CH}_{2} \mathrm{CO}_{2} \mathrm{CH}_{2} \mathrm{CH}_{2}\left(\mathrm{CH}_{2}\right){ }_{11} \mathrm{CH}_{2} \mathrm{CH}_{2} \mathrm{~N}_{3}\right\}$; $3.42\left\{\mathrm{t}, 2 \mathrm{H}, \mathrm{BrCH}_{2} \mathrm{CH}_{2}\left(\mathrm{CH}_{2}\right)_{11} \mathrm{CH}_{2} \mathrm{CH}_{2} \mathrm{CO}_{2} \mathrm{CH}_{2} \mathrm{CH}_{2}\left(\mathrm{CH}_{2}\right)_{11} \mathrm{CH}_{2} \mathrm{CH}_{2} \mathrm{~N}_{3}\right\} ; 3.27\left\{\mathrm{t}, 2 \mathrm{H}, \mathrm{BrCH}_{2} \mathrm{CH}_{2}\left(\mathrm{CH}_{2}\right)_{11}\right.$ $\left.\mathrm{CH}_{2} \mathrm{CH}_{2} \mathrm{CO}_{2} \mathrm{CH}_{2} \mathrm{CH}_{2}\left(\mathrm{CH}_{2}\right)_{11} \mathrm{CH}_{2} \mathrm{CH}_{2} \mathrm{~N}_{3}\right\} ; 2.31 \quad\left\{\mathrm{t}, 2 \mathrm{H}, \mathrm{BrCH}_{2} \mathrm{CH}_{2}\left(\mathrm{CH}_{2}\right)_{11} \mathrm{CH}_{2} \mathrm{CH}_{2} \mathrm{CO}_{2} \mathrm{CH}_{2} \mathrm{CH}_{2}\left(\mathrm{CH}_{2}\right)_{11}\right.$ $\left.\mathrm{CH}_{2} \mathrm{CH}_{2} \mathrm{~N}_{3}\right\}$

\section{PEG550 succinate (14)}

$1.1 \mathrm{~g}$ (11 mmol) freshly prepared succinic anhydride and $2.42 \mathrm{~g}(5 \mathrm{mmol})$ dry PEG550 monomethyl ether were placed in Kugelrohr and reaction was carried out at $130^{\circ} \mathrm{C}$ for $4 \mathrm{~h}$ at 500 mbar pressure. Subsequently excess succinic anhydride was removed under vacuum at $130{ }^{\circ} \mathrm{C}$ in Kugelrohr. 2.60 g PEG550 succinate was obtained (91 \% yield).

${ }^{1} \mathrm{H} N M R\left(\delta, \mathrm{CDCl}_{3}\right): 4.24\left\{\mathrm{t}, 2 \mathrm{H}, \mathrm{CH}_{3} \mathrm{O}\left(\mathrm{CH}_{2} \mathrm{CH}_{2} \mathrm{O}\right)_{\mathrm{n}} \mathrm{CH}_{2} \mathrm{CH}_{2}-\mathrm{O}-\right\} ; 3.63\left\{\mathrm{~m}, 62 \mathrm{H}, \mathrm{CH}_{3} \mathrm{O}-\left(\mathrm{CH}_{2} \mathrm{CH}_{2} \mathrm{O}\right)_{\mathrm{n}^{-}}\right.$ $\left.\mathrm{CH}_{2} \mathrm{CH}_{2} \mathrm{O}-\right\} ; 3.36\left\{\mathrm{~s}, 3 \mathrm{H}, \mathrm{CH}_{3} \mathrm{O}\left(\mathrm{CH}_{2} \mathrm{CH}_{2} \mathrm{O}\right)_{n}-\right\} ; 2.63\left\{\mathrm{~m}, 4 \mathrm{H},-\mathrm{OC}(\mathrm{O})-\mathrm{CH}_{2} \mathrm{CH}_{2}-(\mathrm{O}) \mathrm{CO}-\right\}$

\section{Compound (15)}

$0.738 \mathrm{~g}$ (1.136 mmol) PEG550 succinate (14) and $0.367 \mathrm{~g}$ (4.37 mmol) sodium bicarbonate were taken in $100 \mathrm{~mL}$ round bottom flask. $15 \mathrm{~mL} \mathrm{DMF}$ was added to it and stirred at RT for 10 min. Subsequently $0.5 \mathrm{~g}(0.874 \mathrm{mmol})$ compound 13 was added to it and reaction mixture was stirred at $45^{\circ} \mathrm{C}$ for 3 days. $30 \mathrm{~mL}$ chloroform was added to it and DMF was removed my extraction with $120 \mathrm{~mL}(3 \times 40 \mathrm{~mL}$ ) water. Chloroform containing product was passed through sodium sulfate. Product was obtained by removal of organic solvent. The crude product was purified by reprecipitation from light petroleum ether and methanol. Finally, $0.537 \mathrm{~g}$ waxy solid product was obtained with $53 \%$ yield. 
${ }^{1} \mathrm{H}$ NMR $\left(\delta, \mathrm{CDCl}_{3}\right): 4.22$ (t, $\left.2 \mathrm{H}, \mathrm{PEG}-\mathrm{CH}_{2}-\mathrm{CH}_{2}-\mathrm{O}-\right)$; $4.04\left(\mathrm{~m}, 4 \mathrm{H}, \mathrm{N}_{3} \mathrm{CH}_{2}\left(\mathrm{CH}_{2}\right){ }_{13} \mathrm{CH}_{2} \mathrm{O}-\right.$ and $2 \mathrm{H}$, $\left.\mathrm{N}_{3} \mathrm{CH}_{2}\left(\mathrm{CH}_{2}\right){ }_{13} \mathrm{CH}_{2} \mathrm{CO}_{2} \mathrm{CH}_{2}\left(\mathrm{CH}_{2}\right)_{13} \mathrm{CH}_{2} \mathrm{OCO}\right)$; $3.62\left\{\mathrm{~m}, 36 \mathrm{H}, \mathrm{CH}_{3} \mathrm{O}-\left(\mathrm{CH}_{2} \mathrm{CH}_{2} \mathrm{O}\right)_{\mathrm{n}}-\mathrm{O}-\right\} ; 3.36\{\mathrm{~s}, 3 \mathrm{H}$, $\left.\mathrm{CH}_{3} \mathrm{O}-\left(\mathrm{CH}_{2} \mathrm{CH}_{2} \mathrm{O}\right)_{n}-\mathrm{OH}\right\} ; 3.23\left\{\mathrm{t}, 2 \mathrm{H}, \mathrm{N}_{3} \mathrm{CH}_{2}-\left(\mathrm{CH}_{2}\right)_{8}-\mathrm{CH}_{2} \mathrm{CH}_{2} \mathrm{O}-\mathrm{S}\right\} ; 2.60\left\{\mathrm{~m}, 4 \mathrm{H},-\mathrm{OC}(\mathrm{O})-\mathrm{CH}_{2} \mathrm{CH}_{2}-\right.$ (O)CO-\}; $\left.\left.2.26 \mathrm{~N}_{3} \mathrm{CH}_{2}\left(\mathrm{CH}_{2}\right)_{13} \mathrm{CH}_{2} \mathrm{CO}_{2} \mathrm{CH}_{2}\left(\mathrm{CH}_{2}\right){ }_{13} \mathrm{CH}_{2} \mathrm{OCO}-\right)\right\}$

\section{ChP550-HC-P550}

$0.14 \mathrm{~g}$ (0.194 mmol) compound 16 and $0.243 \mathrm{~g}(0.194 \mathrm{mmol})$ of compound 15 were dissolved in $5 \mathrm{~mL}$ chloroform. $10 \mathrm{~mol} \%$ copper $(\mathrm{I})$ iodide $(3.7 \mathrm{mg})$ and 1 equivalent $(30 \mu \mathrm{L})$ diisopropyl ethylamine (DIPEA) were added to it. The mixture was purged with nitrogen for $15 \mathrm{~min}$, tightly sealed and maintained at $50^{\circ} \mathrm{C}$ for 3 days with continuous stirring. Subsequently, the reaction mixture was centrifuged to remove the copper salt. The linear chromophore containing amphiphilic model compound (ChP550-HC-P550) was purified by reprecipitation (twice) from methanol. Finally, dried under reduced pressure to give around $56 \%$ yield.

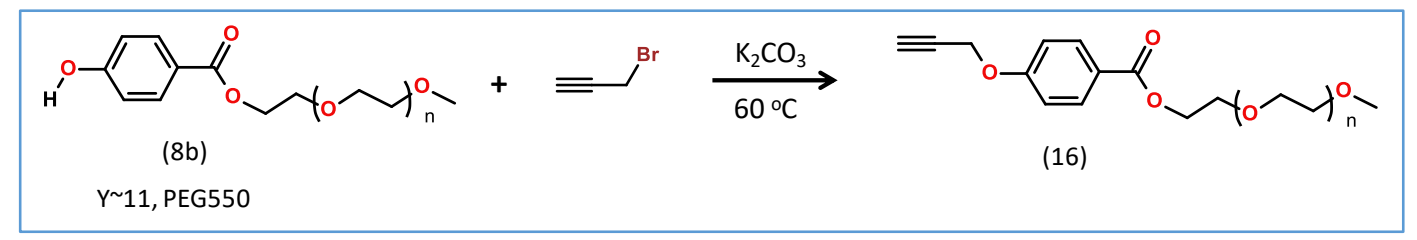

Scheme 6: Synthesis scheme of the compound 16, propargyloxy PEG550 benzoate.

\section{Compound 16}

$1.68 \mathrm{~g}$ compound $8 \mathrm{~b}(2.4 \mathrm{mmol})$ and $1.18 \mathrm{~g}(8.5 \mathrm{mmol})$ potassium carbonate were taken in $100 \mathrm{ml}$ round bottom flask along with $50 \mathrm{ml}$ acetonitrile. $0.8 \mathrm{~g}(6.4 \mathrm{mmol})$ propargyl bromide was added to it. The reaction carried out at $70{ }^{\circ} \mathrm{C}$ for $72 \mathrm{~h}$ under inert atmosphere. Subsequently, acetonitrile was removed, and $100 \mathrm{ml}$ water was added to it. The product was extracted in $150 \mathrm{ml}(3 \times 50 \mathrm{ml})$ ethyl acetate and dried over sodium sulfate. Finally, product was obtained by removing organic solvent under reduced pressure with nearly $76 \%$ yield.

${ }^{1} \mathrm{H} \quad \mathrm{NMR}\left(\delta, \mathrm{ppm}, \mathrm{CDCl}_{3}\right): 8\left\{\mathrm{~d}, 2 \mathrm{H}, \mathrm{HCCCH}_{2} \mathrm{OArCO}_{2}\left(\mathrm{CH}_{2} \mathrm{CH}_{2} \mathrm{O}\right)_{\mathrm{n}} \mathrm{CH}_{3}\right\} ; 6.97\{\mathrm{~d}, 2 \mathrm{H}$, $\left.\mathrm{HCCCH}_{2} \mathrm{OArCO}_{2}-\mathrm{CH}_{2} \mathrm{CH}_{2} \mathrm{O}\left(\mathrm{CH}_{2} \mathrm{CH}_{2} \mathrm{O}\right)_{\mathrm{n}-1} \mathrm{CH}_{3}\right\} ; 4.72\left\{\mathrm{t}, 2 \mathrm{H}, \mathrm{ArCO}_{2}-\mathrm{CH}_{2} \mathrm{CH}_{2} \mathrm{O}\left(\mathrm{CH}_{2} \mathrm{CH}_{2} \mathrm{O}\right)_{\mathrm{n}-1} \mathrm{CH}_{3}\right\}$; 4.42\{d, $\left.2 \mathrm{H}, \mathrm{HCCCH}_{2} \mathrm{OArCO}_{2}\left(\mathrm{CH}_{2} \mathrm{CH}_{2} \mathrm{O}\right)_{\mathrm{n}} \mathrm{CH}_{3}\right\} ; 3.6-3.7\left\{\mathrm{~m}, 3 \mathrm{OH}, \operatorname{ArCO}_{2}-\mathrm{CH}_{2} \mathrm{CH}_{2} \mathrm{O}\left(\mathrm{CH}_{2} \mathrm{CH}_{2} \mathrm{O}\right)_{n-1}\right.$ $\left.\mathrm{CH}_{3}\right\} ; 3.36\left\{\mathrm{~s}, 3 \mathrm{H}, \mathrm{ArCO}_{2}-\mathrm{CH}_{2} \mathrm{CH}_{2} \mathrm{O}\left(\mathrm{CH}_{2} \mathrm{CH}_{2} \mathrm{O}\right)_{\mathrm{n}-1} \mathrm{CH}_{3}\right\} ; 2.54\left\{\mathrm{t}, 1 \mathrm{H}, \mathrm{HCCCH}{ }_{2} \mathrm{OAr} \mathrm{CO}_{2}\left(\mathrm{CH}_{2} \mathrm{CH}_{2} \mathrm{O}\right)_{n}\right.$ $\left.\mathrm{CH}_{3}\right\}$ 


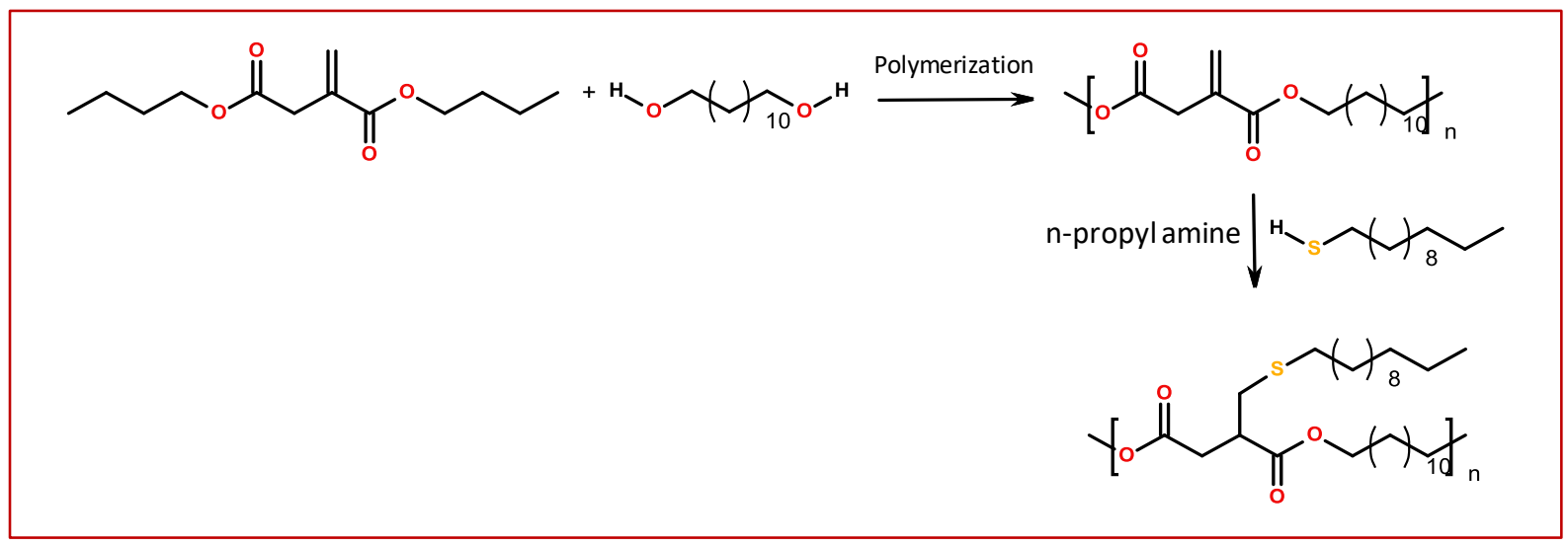

Scheme 7: Synthesis scheme of Dibutyl itaconate and thiol- Michael addition with dodecanethiol

\section{Preparation of Dibutyl itaconate}

A $500 \mathrm{ml}$ round bottom was charged with $20 \mathrm{~g}(0.154 \mathrm{~mol})$ itaconic acid and 192 (2.6 mol) of butanol. $2 \mathrm{~g}$ sulfuric acid was added dropwise to it. The reaction mixture was refluxed for 6 hours and cooled to room temperature, concentrated to about half of its volume and poured into ice water. The product was extracted in chloroform and dried over sodium sulfate and concentrated under reduced pressure. The product was distilled using short path distillation at $150^{\circ} \mathrm{C}(<1$ Torr) and obtained as colorless liquid with overall $70 \%$ yield.

${ }^{1} \mathrm{H}$ NMR (400 MHz, CDCl 3 , $\delta$ ppm): 6.31 (s, $1 \mathrm{H},-\mathrm{CH}_{2} \mathrm{C}(\mathrm{HCH}) \mathrm{COO}-$ ), ; 5.68 (s, $1 \mathrm{H},-\mathrm{CH}_{2}(\mathrm{HCH}) \mathrm{COO}-$ ), $4.15\left(\mathrm{t}, 2 \mathrm{H},-\mathrm{C}\left(\mathrm{CH}_{2}\right) \mathrm{COOCH}_{2}-\right)$, $4.08\left(\mathrm{t}, 2 \mathrm{H},-\mathrm{CH}_{2} \mathrm{COOCH}_{2}-\right)$, 3.32(s, $\left.2 \mathrm{H},-\mathrm{C}\left(\mathrm{CH}_{2}\right) \mathrm{CH}_{2} \mathrm{COO}-\right)$, 1.66$1.56\left(\mathrm{~m}, 4 \mathrm{H},-\mathrm{COOCH}_{2} \mathrm{CH}_{2} \mathrm{CH}_{2} \mathrm{CH}_{3}\right), 1.42-1.33\left(\mathrm{~m}, 4 \mathrm{H},-\mathrm{COOCH}_{2} \mathrm{CH}_{2} \mathrm{CH}_{2} \mathrm{CH}_{3}\right), 0.95-0.91(\mathrm{~m}, 6 \mathrm{H}$, $-\mathrm{COOCH}_{2} \mathrm{CH}_{2} \mathrm{CH}_{2} \mathrm{CH}_{3}$ )

\section{Preparation of poly (dodecyl itaconate)}

$2 \mathrm{~g}(8.26 \mathrm{mmol})$ of dibutyl itaconate, $1.66 \mathrm{~g}(8.26 \mathrm{mmol})$ of 1,12-dodecanediol and $104 \mathrm{mg}$ $(0.166 \mathrm{mmol})$ of dibutyl dilaurate were taken in a reaction vessel. $2.28 \mathrm{mg}(0.02 \mathrm{mmol})$ of $4-$ methylphenol was added as a radical quencher to prevent crosslinking. The polymerization was carried out in oil bath at $120^{\circ} \mathrm{C}$ under nitrogen atmosphere for $8 \mathrm{~h}$ and then in Kugelrohr for $2 \mathrm{~h}$ at $160^{\circ} \mathrm{C}$ under reduced pressure ( $200 \mathrm{mbar}$ ). The polymer was obtained as a white solid after precipitation using chloroform as a solvent and methanol as the non-solvent with $75 \%$ yield. 
${ }^{1} \mathrm{H}$ NMR (400 MHz, CDCl 3 , $\delta$ ppm): 6.36 (s, $\left.1 \mathrm{H},-\mathrm{CH}_{2} \mathrm{C}(\mathrm{HCH}) \mathrm{COO}-\right)$, 5.73 (s, $1 \mathrm{H},-\mathrm{CH}_{2}(\mathrm{HCH}) \mathrm{COO}-$ ), $4.17\left(\mathrm{t}, 2 \mathrm{H},-\mathrm{C}\left(\mathrm{CH}_{2}\right) \mathrm{COOCH}_{2}-\right), 4.10\left(\mathrm{t}, 2 \mathrm{H},-\mathrm{CH}_{2} \mathrm{COOCH}_{2}-\right), 3.37\left(\mathrm{~s}, 2 \mathrm{H},-\mathrm{C}\left(\mathrm{CH}_{2}\right) \mathrm{CH}_{2} \mathrm{COO}-\right), 1.68$ (m, $\left.4 \mathrm{H},-\mathrm{OCOCH} \mathrm{CH}_{2}\left(\mathrm{CH}_{2}\right)_{16} \mathrm{CH}_{2} \mathrm{CH}_{2} \mathrm{OCO}-\right), 1.29\left(\mathrm{~m}, 16 \mathrm{H},-\mathrm{OCOCH}_{2} \mathrm{CH}_{2}\left(\mathrm{CH}_{2}\right)_{8} \mathrm{CH}_{2} \mathrm{CH}_{2} \mathrm{OCO}-\right)$

Post-polymerisation modification of poly(dodecyl itaconate) by Michael addition with dodecanethiol

$500 \mathrm{mg}$ (1.68 mmol, w.r.t repeat unit) poly(dodecylitaconate) and $681 \mathrm{mg}$ (3.36 mmol) of dodecane thiol was taken in a reaction vessel along with $3 \mathrm{ml}$ dry chloroform. Catalytic amount ( 2 drops) of n-propyl amine was added to it and purged with nitrogen. The reaction was carried out at room temperature under nitrogen environment. The aliquots was after every $6 \mathrm{~h}$ and precipitated in methanol (non-solvent). NMR and GPC of the aliquots were recorded. The reaction was completed in $24 \mathrm{hrs}$. 


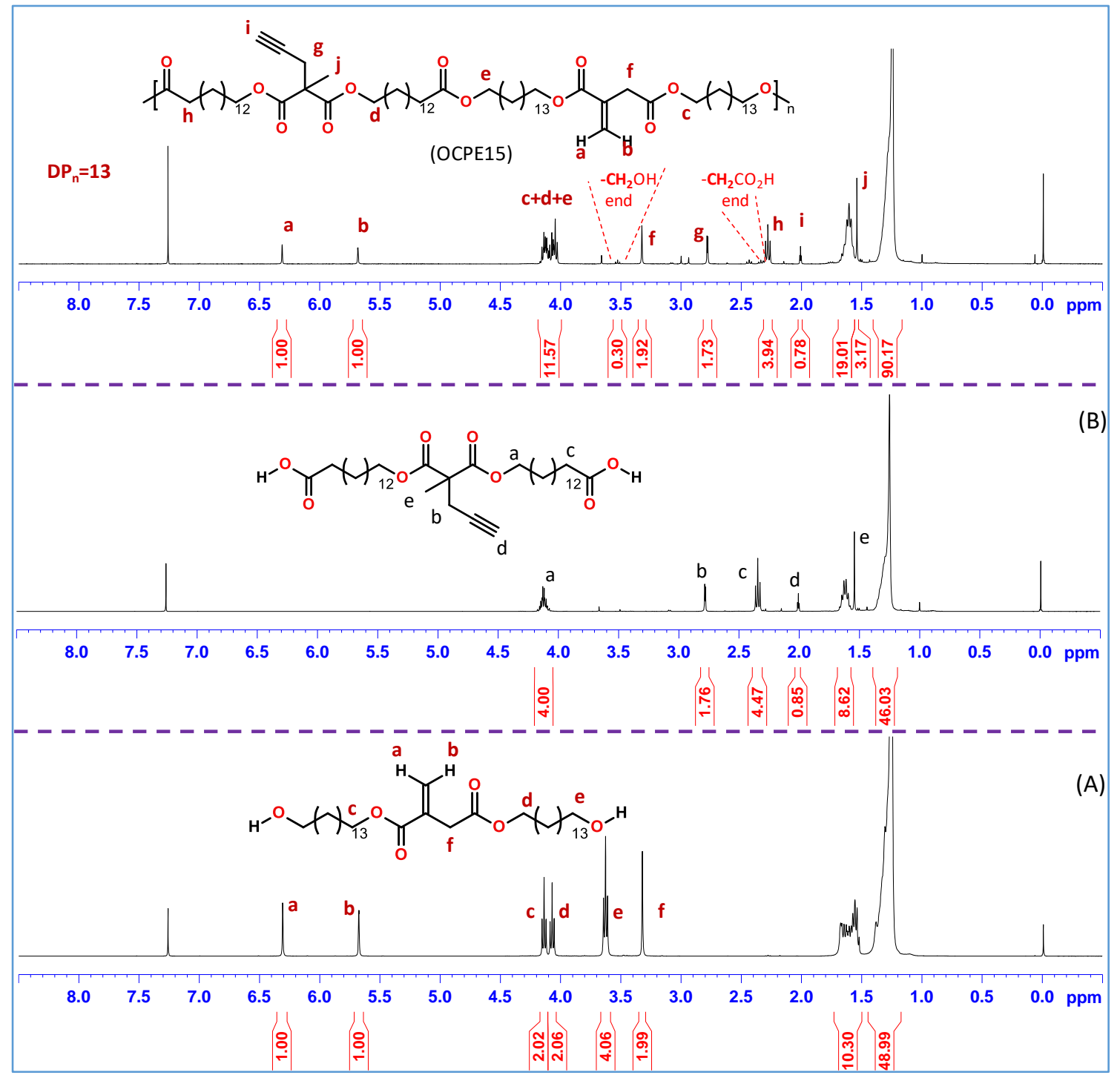

Figure S1: ${ }^{1} \mathrm{H}$ NMR spectrum of itaconate bearing diol (A), propargyl bearing di-acid (B) and parent polymer (OCPE15). Intensities of the peaks are accordance to the structures. 


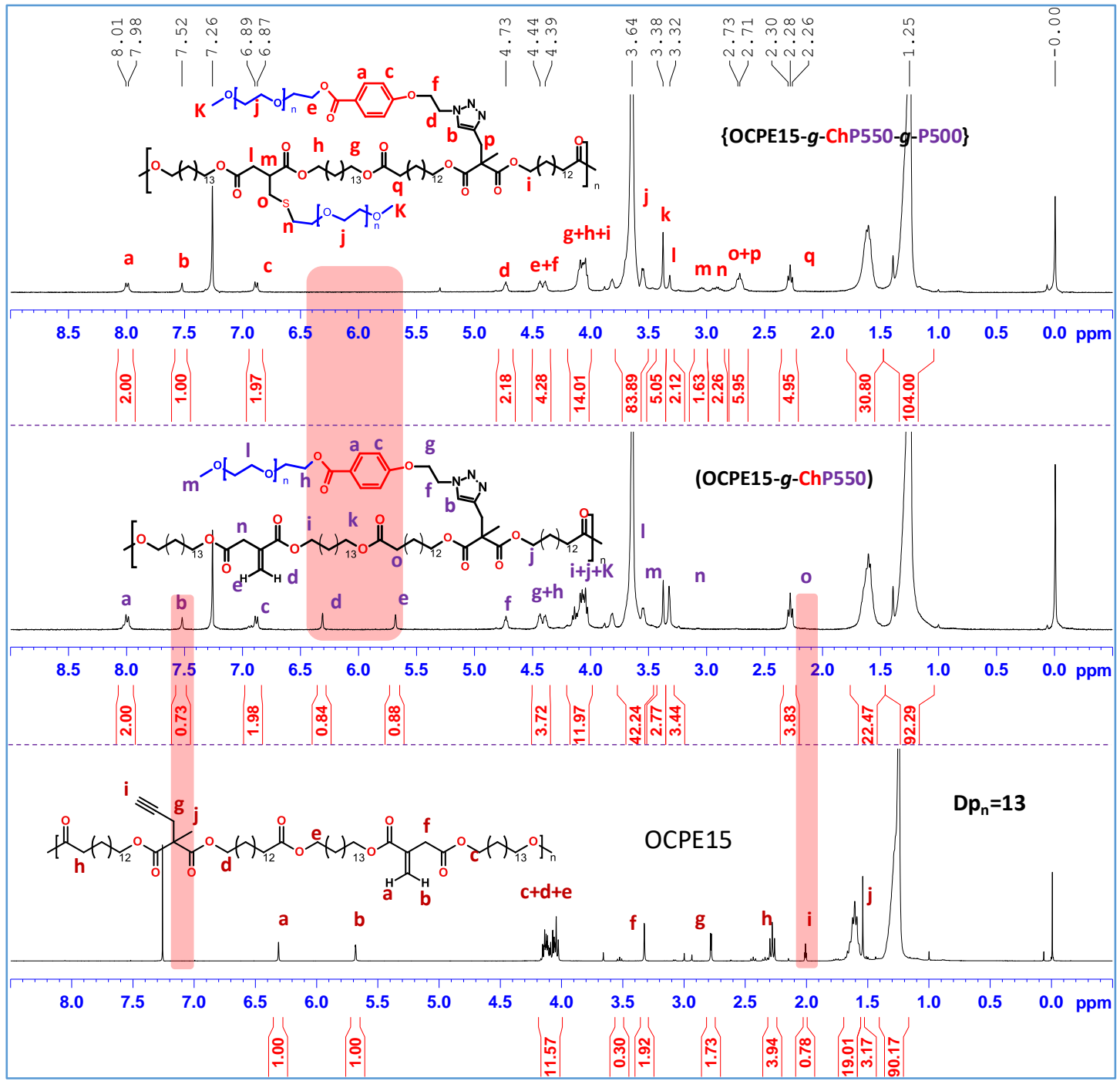

Figure S2: Stacked ${ }^{1} H$ NMR spectrum of parent (OCPE15) and those after grafting chromophore bearing PEG550 (OCPE15-g-ChP550) and PEG550 thiol segment (OCPE15-g-ChP550-g-P550). Disappearance of propargyl peak and itaconate peaks are highlighted. Degree of polymerization estimated from the intensity ratio of itaconate peak $(a, 6.31 \mathrm{ppm})$ and end $-\mathrm{CH}_{2} \mathrm{OH}$ peak at $3.63 \mathrm{ppm}$. 


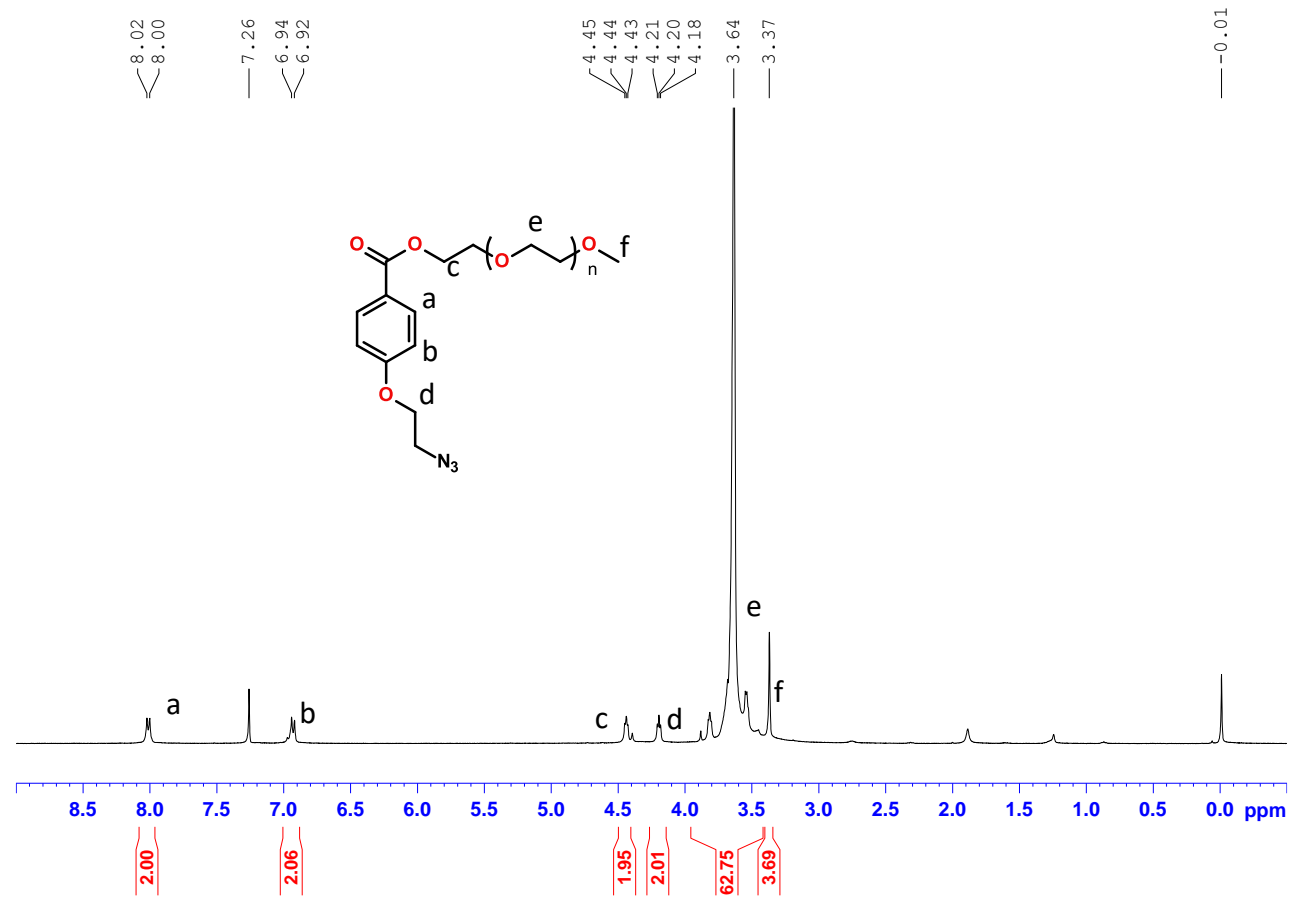

Figure S3: ${ }^{1} \mathrm{H}$ NMR spectrum of chromophore bearing PEG550 azide (10 b)

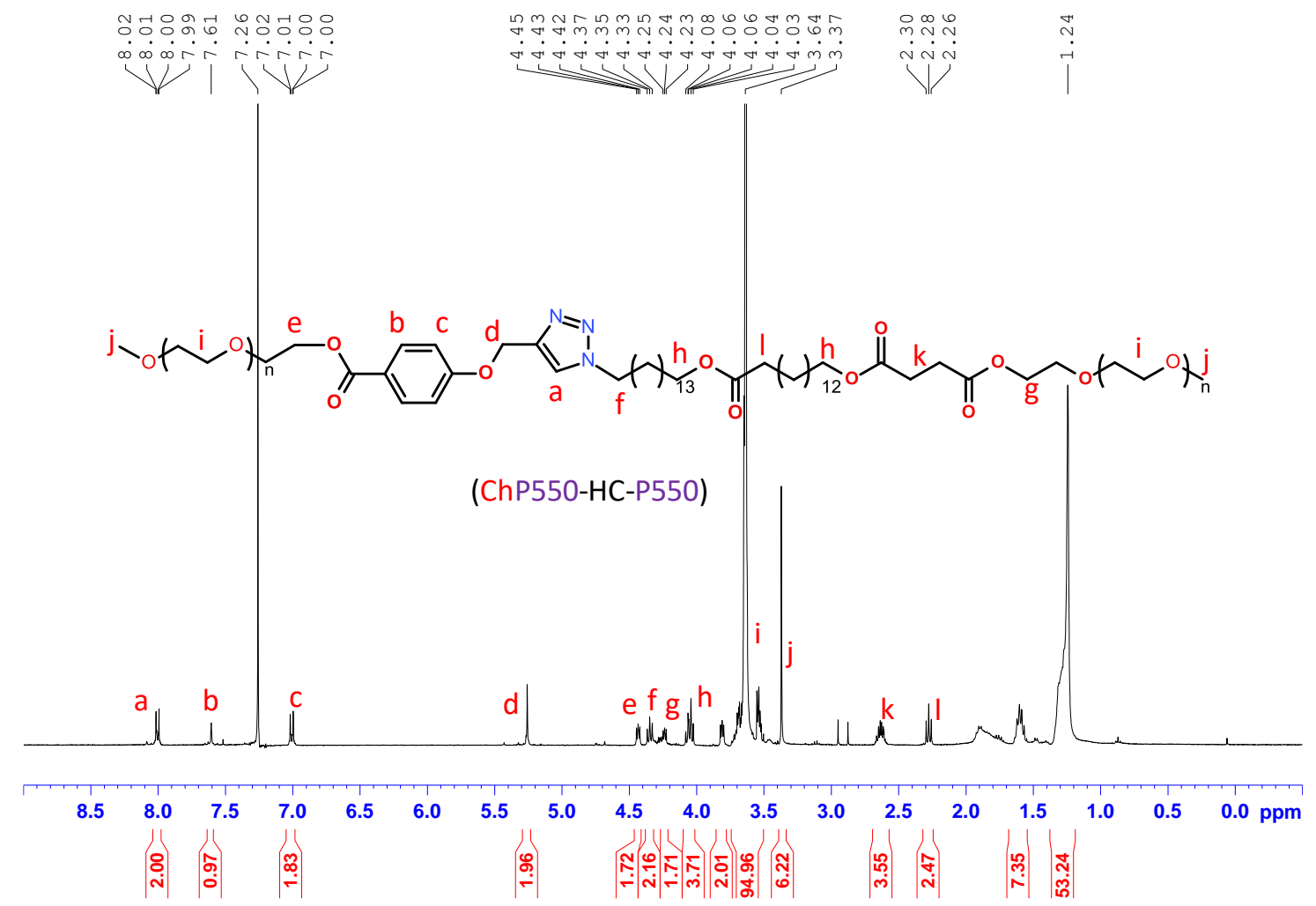

Figure S4: ${ }^{1} H$ NMR spectrum of chromophore-bearing bola-amphiphilic model compound (ChP550HC-P550) 


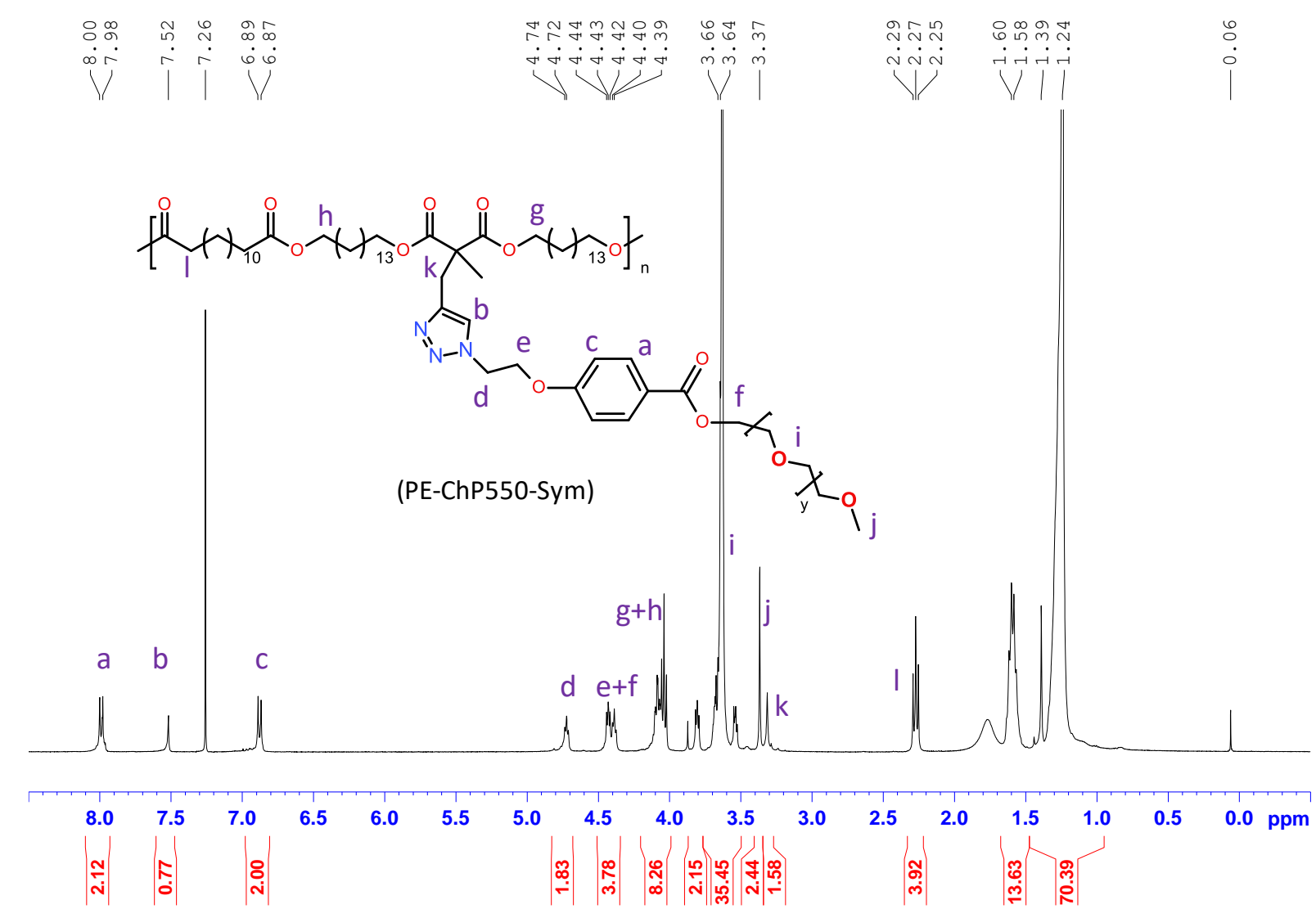

Figure S5: ${ }^{1} H$ NMR spectrum of chromophore-bearing symmetric polymeric model compound (PE-gChP550-Sym) 

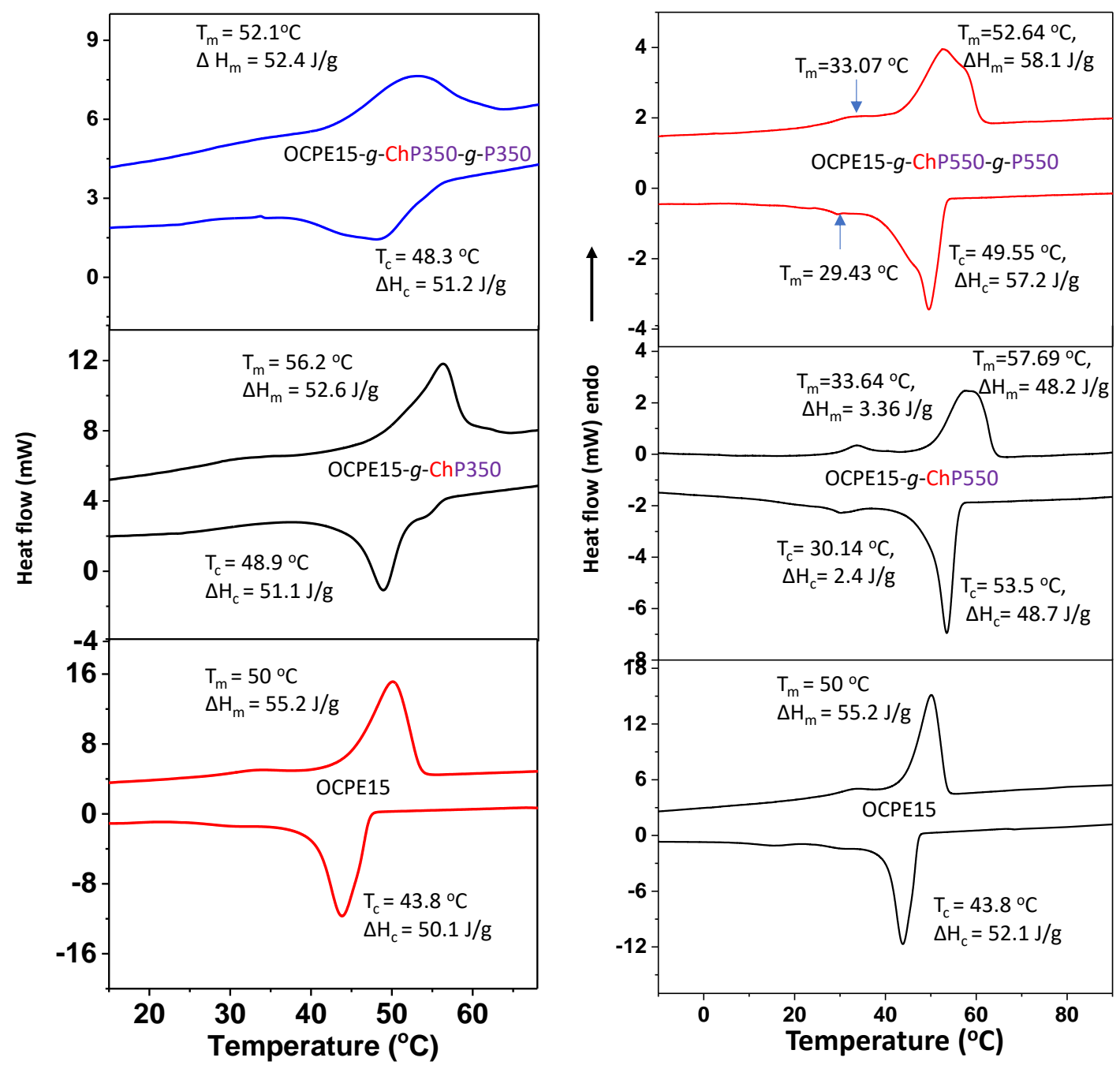

Figure S6: DSC of the parent polymer, OCPE15 and PEG (350 and 550) grafted copolymers, namely OCPE15-g-ChP350 and OCPE15-g-ChP550, OCPE15-g-ChP350-g-P350 and OCPE15-g-ChP550-g-P550. The single exotherm/endotherm corresponds to melting/crystallization of backbone hydrocarbon segment. 


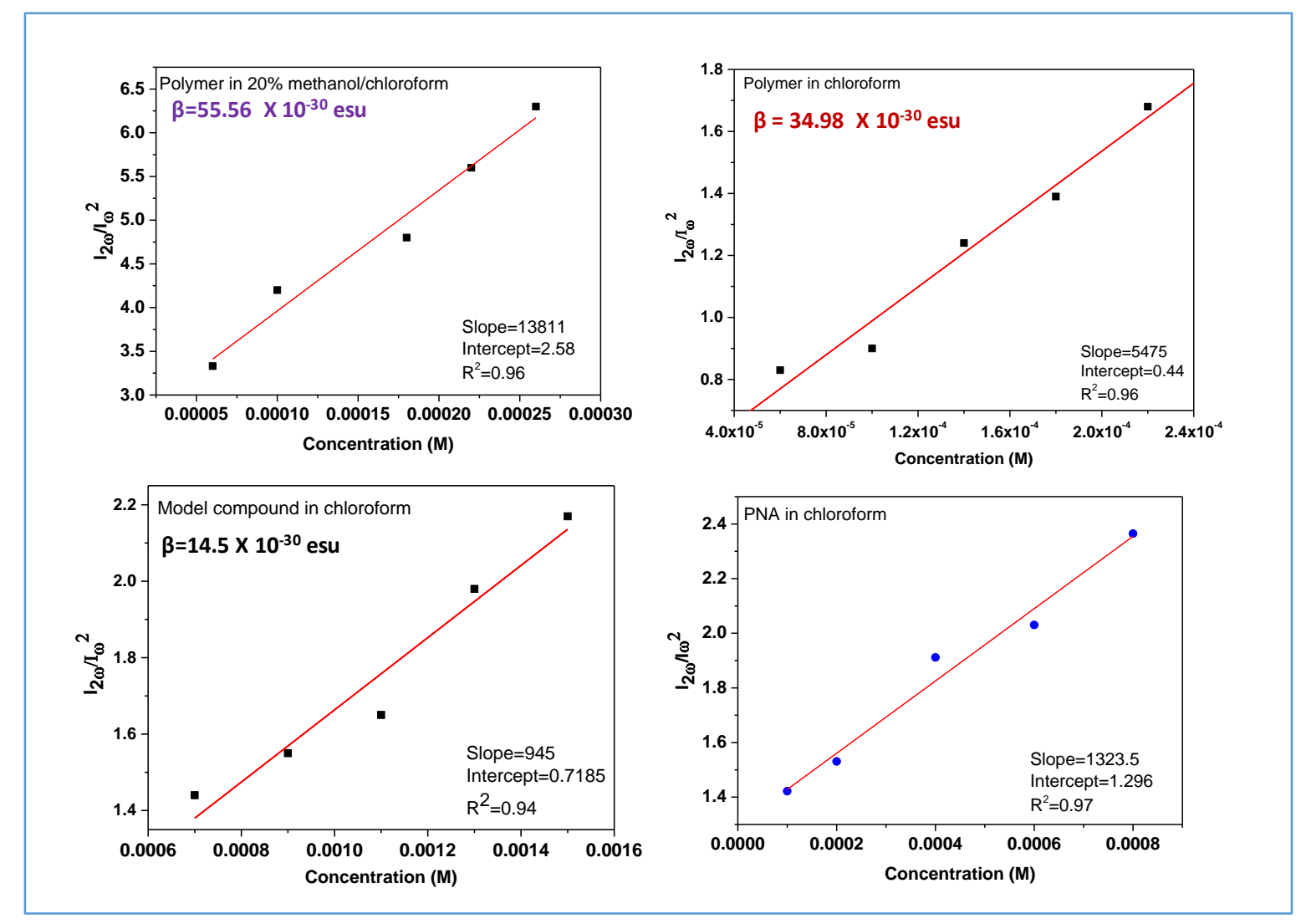

Figure S7: Variation of normalized I $2 \omega$ for polymer, OCPE15-g-ChP550-g-P550, model compound and p-nitroaniline (PNA), as a function of their concentration; in the polymer case, concentration is with respect to the repeat unit, which also reflects the concentration of the chromophore. The $\beta_{P N A}$ was in both chloroform and $20 \%$ methanol/chloroform were found to be almost the same; hence, the $\beta_{\text {polymer }}$ in $20 \%$ methanol/chloroform mixture was estimated based on $\beta_{P N A}$ in pure chloroform. $\beta$ is estimated using the equation, $B_{\text {sample }}=\beta_{P N A}$ (slope of sample/slope of PNA) $)^{0.5}$.

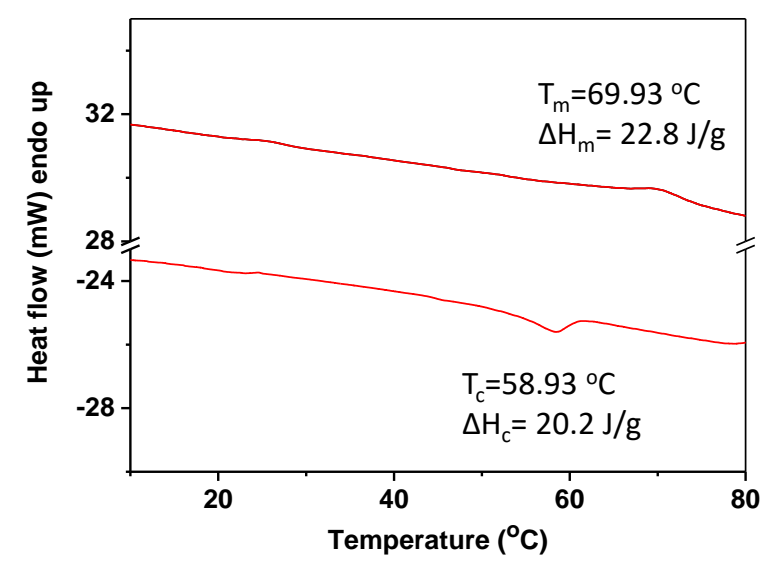

Figure S8: DSC of OCPE15-g-ChP550-g-P550 in water; the solution was prepared by adding a concentrated THF solution of the polymer $(1 \mathrm{mg} / \mathrm{ml})$ into water and warming the solution to $50{ }^{\circ} \mathrm{C}$ and sonicated to allow evaporation of the THF. The exothermic and endothermic peaks signify melting and crystallization of backbone $\mathrm{HC}$. 
Evolution of molecular weight during thiol-Michael addition

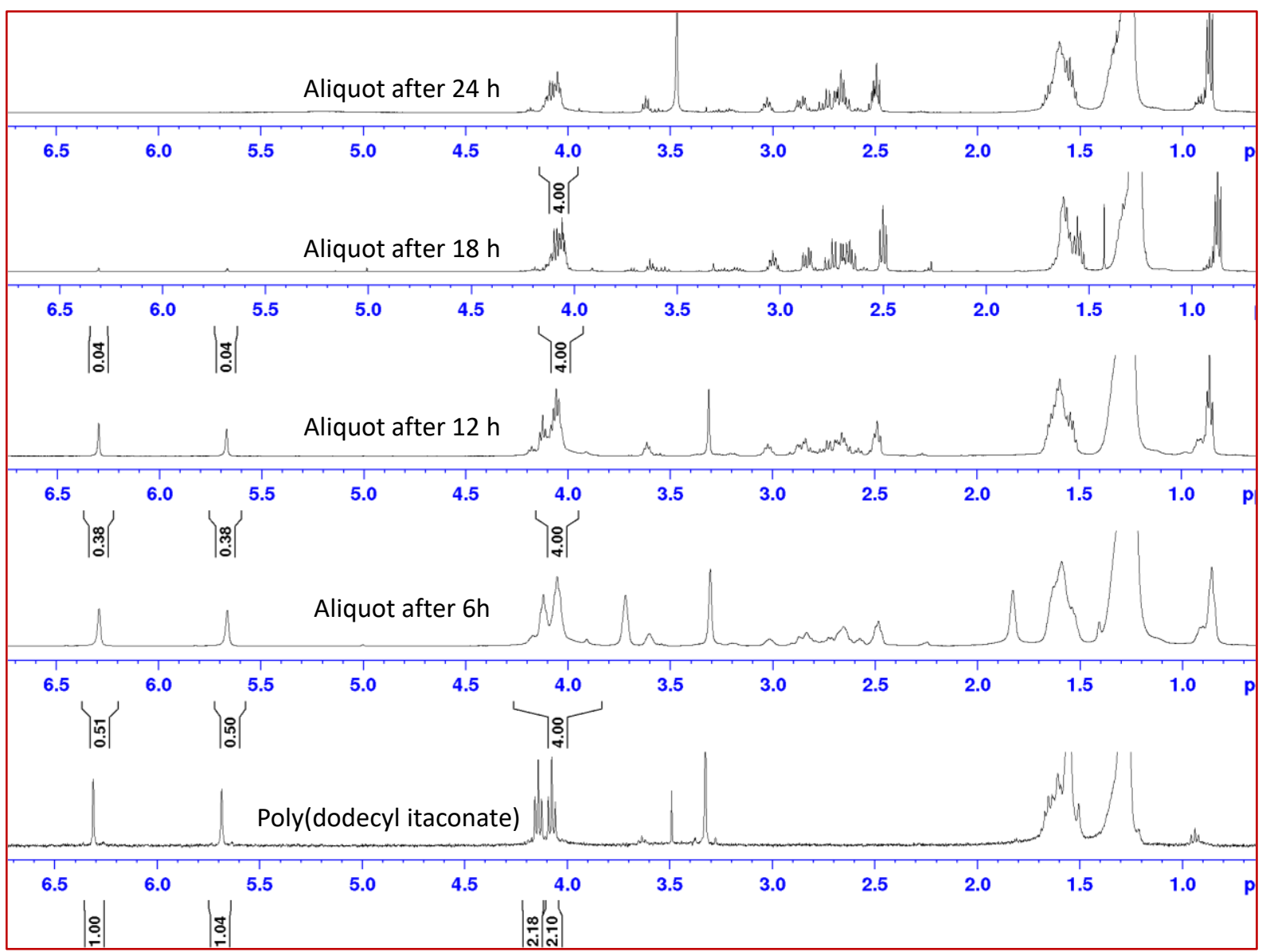

Figure S 9: ${ }^{1} \mathrm{H}$ NMR spectrum of the parent poly (dodecyl itaconate) and thiol-Michael reaction aliquots of parent polymer and dodecane thiol. The aliquots were taken at specific time interval and precipitated in methanol before NMR measurements. The thiol-Michael was completed in $24 \mathrm{~h}$

\begin{tabular}{|c|c|c|}
\hline Polymer & Mn (Da) & Mw (Da) \\
\hline Parent polymer & 5465 & 12887 \\
\hline Aliquot after 6 h & 6333 & 16616 \\
\hline Aliquot after 12 h & 6772 & 19729 \\
\hline Aliquot after 18 h & 7218 & 33169 \\
\hline Aliquot after $24 \mathrm{~h}$ & 7446 & 54867 \\
\hline
\end{tabular}

Table S1: The table shows GPC molecular of the parent polymer and thiol-Michael reaction aliquots of parent polymer and dodecane thiol. The aliquots were taken at specific time interval and precipitated in methanol before GPC measurements. An increment in molecular weight with process of the thiolMichael reaction is noticed. The thiol-Michael was completed in $24 \mathrm{~h}$. 


\begin{tabular}{|c|c|c|c|}
\hline Polymer & $\mathbf{M}_{\mathbf{n}}$ & $\mathbf{M}_{\mathbf{w}}$ & PDI \\
\hline OCPE15 & 10700 & 14950 & 1.48 \\
\hline OCPE15-g-ChP550 & 14706 & 25860 & 1.75 \\
\hline $\begin{array}{c}\text { OCPE15-g-ChP550- } \\
g-P 550\end{array}$ & 7800 & 10600 & 1.35 \\
\hline PE & 10500 & 14700 & 1.4 \\
\hline PE-g-ChP550-sym & 15256 & 21930 & 1.44 \\
\hline
\end{tabular}

Table S2: Table summarizing the molecular weight of parent and graft copolymers. An unusual reduction in molecular weight was seen for OCPE15-g-ChP550-g-P550. Similar observation was also noticed for our previously reported hetero grafted amphiphilic copolymer system, which was attributed to the adaptation of more compact conformation by the amphiphilic polymer, thereby leading to a reduction in the hydrodynamic size and an apparent decrease in the $M_{n}$ value determined by GPC. ${ }^{1}$

\section{Estimation of $f$ (local field factor)}

$\beta_{\text {polymer }}=n_{c} f \beta_{\text {chromophore, }}$

$n_{\mathrm{c}}$ is the average number of chromophores in the polymer chain, which is 6 , as determined from NMR and $f$ is a local field factor that takes into account electrostatic effects due to neighbouring chromophores at close proximity.

Here, $\beta_{\text {chromophore }}=14.5 \times 10^{-30}$ esu and $\beta_{\text {polymer }}=55.5 \times 10^{-30}$ esu

Hence $f=\{55.5 /(14.5 \times 6)\}=0.638$

\section{References}

1. Sarkar, R.; Gowd, E. B.; Ramakrishnan, S., De-symmetrizing periodically grafted amphiphilic copolymers: design, synthesis and generation of Janus folded chains. Polymer Chemistry 2019, 10 (14), 1730-1740.

2. Sarkar, R.; Gowd, E. B.; Ramakrishnan, S., Precise control of grafting density in periodically grafted amphiphilic copolymers: an alternate strategy to fine-tune the lamellar spacing in the sub-10 nm regime. Polymer Chemistry 2020, 11 (25), 4143-4154. 